\title{
Theoretische Überlegungen zu Fremd- und Selbstführung
}

»Aber das heißt, wir sind auch Gefährder, oder wie?«, fragt Hassan M. in der dritten Gruppendiskussion besorgt in die Runde. Die Unkenntnis darüber, nach welchen Kriterien der polizeiliche Fachbegriff operiert und wie sicherheitspolitisch zwischen sguten Muslim:innen unterschieden wird, führt zu einem generellen Unbehagen unter den Diskussionsteilnehmer:innen. Sie befürchten, als Bedrohung wahrgenommen zu werden, zum Beispiel weil sie regelmäßig Moscheen besuchen oder in religiösen Vereinen aktiv sind. Von strafrechtlicher Relevanz sind diese Aspekte des alltäglichen Lebens weit entfernt, das ist allen bewusst. Gleichzeitig kommen sie nicht umhin, sich zu fragen, was passieren könnte, wenn bestimmte Orte und Akteur:innen mit Salafismus und Radikalisierung in Verbindung gebracht werden oder sie mit Gruppen und Personen assoziiert werden, die dem Spektrum des sogenannten politischen Islams zugeordnet werden. Die muslimischen Diskussionsteilnehmer:innen fühlen sich unter Druck gesetzt, ihre Loyalität und Zugehörigkeit unter Beweis zu stellen, um einer möglichen Zuordnung in eine problematische Kategorie vorzubeugen. Allein der Gedanke, als Gefährder:in, Salafist:in oder Terrorist:in wahrgenommen zu werden, führt dazu, dass sie selbst ihre Körper, ihre Sprache und ihre Kleidung, ihr Verhalten und ihre Mobilität im öffentlichen Raum daraufhin befragen, wie sie wahrgenommen werden und wie sie sich den diskursiven Anforderungen an eine sgute Bürgerschaft‘ anpassen oder entziehen. 
Die Angst davor, als gefährlich eingeordnet zu werden, kann auch dazu führen, anderen Muslim:innen und muslimischen Akteur:innen gegenüber mit Vorsicht zu begegnen. Im Alltag bedeutet das, Kontaktschuldvorwürfen ${ }^{1}$ vorzubeugen und daher Gruppen und Individuen, die möglicherweise als islamistisch eingestuft werden, zu meiden. Es bedeutet auch, sich zu überlegen, ob und gegebenenfalls in welcher Weise sie Stellung beziehen $\mathrm{zu}$ den Themen Terrorismus, Salafismus und Gewalt, die im Namen des Islams gerechtfertigt werden. ${ }^{2}$ Weder Strafe und Zwangsmaßnahmen noch direkte staatliche Kontrolle und Disziplinierung engen in dieser Sequenz die Diskussionsteilnehmer:innen im Kontext des Bedrohungsszenarios des islamistischen Terrorismus ein. Vielmehr tun dies Andeutungen und Erwartungen, Befürchtungen und exemplarische Erfahrungen Anderer, mediale und politische Diskursregeln, die sie als Muslim:innen homogenisieren und als Kollektiv als (potentielle) Gefahr adressieren. In ihren Selbstbildern und Verhaltensweisen tritt jene Machttechnologie in Erscheinung, die Foucault als Gouvernementalität ${ }^{3}$ (gouverner: regieren; mentalité: Denkweise) bezeichnet.

\section{Gouvernementalität, Biopolitik und Rassismus}

Dabei handelt es sich um eine Regierungstechnologie, »die aus den Institutionen, den Vorgängen, Analysen und Reflexionen, den Berechnungen und den Taktiken gebildete Gesamtheit, welche es erlauben, diese recht spezifische, wenn auch sehr komplexe Form der Macht auszuüben, die als Hauptzielscheibe die Bevölkerung, als wichtigste Wissensform die politische Ökonomie und als wesentliches technisches Instrument die Sicherheitsdispositive hat«. ${ }^{4}$ Im gegenwärtigen Sicherheits-

\footnotetext{
1 Schiffauer 2020.

2 Die genannte Sequenz wird im Kapitel Ver_Handlungen im Abschnitt Ent_Solidarisierung rekonstruiert.

3 Foucault 2017a.

4 Ebd.: 162.
} 
diskurs steht der nach Foucault üblichen Disziplinarmacht, die (islamistischen Terrorismus) verbietet, (Gefährder:innen) beobachtet und (islamistische Straftäter:innen) diszipliniert, eine andere Technik zur Seite, die den Rest der Muslim:innen kollektiv zur Selbstführung anhält. Foucaults Ausführungen zur Gouvernementalität sind für die vorliegende Untersuchung instruktiv, weil sie den Blick auf Sicherheitsdispositive lenken, deren Funktion darin liegt, "auf eine Realität zu antworten, so daß diese Antwort jene Realität aufhebt, auf die sie antwortet«. ${ }^{5}$ Im Verhältnis von Sicherheit und antimuslimischem Rassismus ist das Ziel einer gouvernementalen Führung, indirekt auf die Bevölkerung einzuwirken, also »auf die der Bevölkerung offensichtlich entfernten Dinge Einfluß [zu] nehmen ${ }^{6}{ }^{6}$

Bublitz zufolge handelt es sich bei der Gouvernementalität um »eine spezifische Form der Biomacht, in der das Leben des Menschen als Lebewesen auf dem Spiel steht und Gegenstand wissens- und technikbasierter >Praktiken des Selbst « wird «. ${ }^{7}$ Die Biopolitik, die Foucault bereits in Der Wille zum Wissen. Sexualität und Wahrheit I untersucht, nimmt seit dem 18. Jahrhundert die Bevölkerung - unter dem Vorwand innere und äußere Gefahren abwehren zu wollen - ins Visier biologischer Umgestaltung. Sie löst das alte Souveränitätsprinzip »sterben zu machen oder leben zu lassen « ab durch die Regel »leben zu machen oder in den Tod zu stoßen $\varkappa^{8}$ Die Biopolitik zielt auf die »vollständige Durchsetzung des Lebens «. ${ }^{9}$ Sie befragt Gesundheit, Sterblichkeit, Geburtenziffer, Hygiene und Lebensdauer der Bevölkerung. Ihre Geburtsstunde fällt nicht zufällig zusammen mit der

»Entwicklung eines spezifischen politischen Wissens und neuer Disziplinen wie Statistik, Demografie, Epidemiologie und Biologie, die Lebensprozesse auf dem Niveau von Bevölkerungen analysieren, um

\footnotetext{
5 Ebd.: 76.

6 Ebd.: 111.

7 Bublitz 2014: 94.

8 Foucault 2017b: 134.

9 Ebd.: 135.
} 
Individuen und Kollektive mittels korrigierender, exkludierender, normalisierender, disziplinierender, therapierender oder optimierender Maßnahmen zu >regieren « ${ }^{10}$

Als Beispiel besonders geeignet ist die sich im 18. Jahrhundert in Deutschland etablierende Polizeiwissenschaft, die, so Foucault, »eine vom Prinzip der Staatsräson beherrschte Regierungstechnologie angenommen « hat, indem sie sich den »Problemen der Population« annimmt, um die Bevölkerung »für die Stärke des Staates« $\mathrm{zu}$ >verbessern ${ }^{11}$ Das macht die Biopolitik zum Dreh- und Angelpunkt einer "Macht-Technologie, deren Gegenstand und Ziel das Leben ist $\aleph^{12}$, insofern sie (bestimmtes) Leben aufwertet, verlängert und vervielfacht. Sarasin ${ }^{13}$ hebt diesen Aspekt ausdrücklich hervor: Anders als es etwa in Agambens Souveränitätstheorie zum Tragen kommt, die sich unter dem Begriff der Biopolitik auf das Töten beschränkt, kreist Foucaults Konzept »um die Produktion, nicht um die Tötung von Leben «. ${ }^{14}$ Für Foucault stellt sich deshalb die Frage danach, wie eine Macht, die Leben maximieren und vervielfältigen will, töten kann: »Wie sollte eine Macht ihr höchstes Vorrecht in der Verhängung des Todes äußern, wenn ihre Hauptaufgabe darin besteht, das Leben $\mathrm{zu}$ sichern, $\mathrm{zu}$ verteidigen, $\mathrm{zu}$ stärken, zu mehren und zu ordnen? $^{15}$ Hier kommt der Rassismus ins Spiel.

Tatsächlich hängen Foucaults Überlegungen zur Biopolitik unmittelbar mit jenen zum modernen Rassismus zusammen: Der Rassismus legitimiert im Namen der Biopolitik das Töten. In seinem Vortrag In Verteidigung der Gesellschaft betont Foucault, dass die Biopolitik nicht ohne den Rassismus gedacht werden kann, Rassismus stellt eine intrinsische Komponente innerhalb des Machtapparates dar und ist das "Mittel, um in diesen Bereich des Lebens, den die Macht in Beschlag genommen

\footnotetext{
10 Lemke 2007: 14.

11 Foucault 2013a: 181.

12 Foucault 2001: 300.

13 Sarasin 2003: 58.

14 Ebd.: 61.

15 Foucault 2017b: 133.
} 
hat, eine Zäsur einzuführen: die Zäsur zwischen dem, was leben, und dem, was sterben muß «. ${ }^{16}$ Rassismus ermöglicht es, die Bevölkerung $\mathrm{zu}$ fragmentieren, $\mathrm{zu}$ disziplinieren und Gruppen gegeneinander auszuspielen, indem >Rassen ^produziert und als höherwertig bzw. minderwertig hierarchisiert werden. Wichtig dabei ist, dass Foucault unter dem Recht zu töten weit mehr versteht als den direkten Mord. Im Gegenteil zählen darunter auch indirekte Formen, wie »jemanden der Gefahr des Todes ausliefern, für bestimmte Leute das Todesrisiko oder ganz einfach den politischen Tod, die Vertreibung, Abschiebung usw. erhöhen ${ }^{17}{ }^{17}$

Zwar beschränkt sich Foucault in seinem Vortrag in erster Linie auf einen biologistischen Rassismus, doch kann, wie Rose anmerkt, ein ähnliches Prinzip ebenso im Falle eines kulturell argumentierenden Rassismus beobachtet werden. ${ }^{18}$ Mit Balibar und Hall ${ }^{19}$ versteht Rose Rassismus

»als machtvolles Operations- und Differenzierungsmodell [...], das in das Kontinuum einer Bevölkerung bedeutsame Unterscheidungen wie seigen oder >fremd durch diejenigen, für die die letztgenannten Kategorien in Anschlag gebracht werden, stärker als erstere einer Gefahr ausgesetzt sind «. ${ }^{20}$

Stoler kritisiert, dass in Foucaults enger Rassismus-Analyse, ausgehend von seiner Geschichte der Sexualität, der Kolonialismus zu kurz kommt. Schließlich spielen auch imperiale Schauplätze außerhalb Europas eine Rolle in der Produktion europäischer Diskurse um Sexualität, weshalb Foucault »die Praktiken [übergeht], welche eine Rassenterminologie in den Körper einschreiben ${ }^{21}{ }^{21}$ Bürgerliche Identitäten in europäischen Metropolen und in >ihren Kolonien in Übersee

\footnotetext{
16 Foucault 2001: 301.

17 Ebd.: 303.

18 Rose 2015: 334.

19 Balibar 1992; Hall 1994.

20 Rose 2015: 334.

21 Stoler 2002: 307.
} 
waren »insgeheim nachhaltig durch Rassenvorstellungen codiert «. ${ }^{22}$ Stoler hinterfragt, weshalb Foucault die Geburtsstunde des Rassismus ins späte 19. Jahrhundert legt, obgleich sich das Phänomen historisch, sozial und politisch breiter auffächern lässt. ${ }^{23}$ Auch Lemke vermisst die Berücksichtigung des Kolonialismus und die spezifischen Intersektionen zur Sexualität in Foucaults Kritik des Rassismus. Gleichzeitig geht er davon aus, dass dies analytische Vorteile bietet, indem Rassismus weder als "Ausnahmesituation oder Antwort auf soziale Krisen « ${ }^{24}$ noch als individuelle Handlung Einzelner verharmlost wird. Im Anschluss an Foucault kann Rassismus verstanden werden als Gesellschaft strukturierend, Politik leitend und institutionell manifestiert.

In Foucaults Überlegungen zur Gouvernementalität wird der Begriff der Biopolitik nicht mehr verwendet. In seiner weiteren Auseinandersetzung mit dem Problem der Bevölkerungsregierung spielt auch die Sexualität keine prominente Rolle mehr. Möglicherweise ist das der Grund, weshalb Foucault fortan dem Rassismus keine Beachtung mehr schenkt. Letztendlich hängt sie in seinen Überlegungen zur Biopolitik noch eng mit der sexuellen, und damit der biologischen Regulierung und (Um-)Gestaltung der Bevölkerung zusammen. Sarasin geht davon aus, dass Foucaults Konzept der Biopolitik dennoch »ein Torso mit vielen Schnittstellen für mögliche theoretische Weiterentwicklungen unter dem Stichwort >Gouvernementalität« « ist. ${ }^{25}$ Dies trifft gewiss für die Untersuchung des gegenwärtigen Rassismus unter dem Gesichtspunkt der Sicherheitsdispositive zu. Angesichts der Verquickung von (disziplinierender) Macht mit (normalisierenden) Selbsttechnologien stellt sich die Frage, wie sich rassistische Praktiken transformieren und wie das Subjekt zur Selbstführung angeleitet wird, wenn die >Freiheit und die >Sicherheit der Subjekte zur raison d'être liberaler Regierungspraxis werden. Die gouvernementale Macht setzt »in einer fundamentalen Weise nicht einfach die disziplinäre Anpassung und Unterwerfung

\footnotetext{
22 Ebd.: 308.

23 Ebd.: 312.

24 Lemke 2007: 59.

25 Sarasin 2005: 177.
} 
der Individuen voraus [...], sondern ganz im Gegenteil deren Freiheit «. ${ }^{26}$ Sicherheitstechnologien »fabrizieren eine Freiheit, die permanent bedroht und gefährdet ist und daher zur Grundlage immer neuer Interventionen wird, die einem Sicherheitskalkül der Gesellschaft folgen «. ${ }^{27}$ Doch was bedeuten Freiheit und Sicherheit im antimuslimischen Rassismus für die Führung rassifizierter Subjekte?

\section{Freiheit und (Selbst-)Führung im (antimuslimischen) Rassismus}

»Regieren heißt, die Dinge regieren ${ }^{28}$, schreibt Foucault. Das funktioniert über Erziehung, Kampagnen und Überzeugungsarbeit, um Einfluss auf die Gewohnheiten der Bevölkerung zu nehmen - auf ihre Befürchtungen, darauf, wie sie sich bewegen und verhalten, wie sie leben und denken. Auch mithilfe von Rassismus kann dieser Regel Folge geleistet werden. Rassismus legitimiert das Strafen, Ausschließen und Kriminalisieren, doch genauso gut können im Rassismus auch Einbezug, >Integration und Vereinnahmung funktional greifen, und das ohne die eigentliche Zielsetzung, nämlich rassifizierte Subjekte unterzuordnen, aus dem Blick zu verlieren.

Mecheril spricht von einem »antizipierten Rassismus « ${ }^{29}$, um $\mathrm{zu}$ beschreiben, dass Rassismus auch erfahren wird, indem man Geringschätzung und Degradierung vorwegnimmt oder Angriffe und Attacken befürchten muss. Rassifizierte Personen können deswegen Wut, Angst, Verzweiflung oder auch Scham empfinden. Mecheril beschreibt Vorsicht bis hin zur Vermeidung von Kontakten und dem Rückzug aus bestimmten Situationen als Reaktionen auf Rassismuserfahrungen.

Nach der Bedeutung des Sicherheitsdiskurses gefragt berichten unsere Gesprächspartner:innen von ihren Gefühlen angesichts der Alltäg-

$\begin{array}{ll}26 & \text { Ebd.: } 184 . \\ 27 & \text { Bublitz 2014: } 95 . \\ 28 & \text { Foucault 2017a: } 147 . \\ 29 & \text { Mecheril 1994: 60. }\end{array}$


lichkeit ihrer Rassismuserfahrungen. ${ }^{30}$ Ahmed diskutiert Rassismus im Zusammenhang mit einer "gewisse[n] paranoide[n] Sorge um Dinge, die bereits passieren und passieren könnten «, sodass sich rassifizierte Menschen als Effekt von Rassismuserfahrungen vorbeugend selbst zensieren und zurückziehen. ${ }^{31}$ Rassismus, Sicherheit und Gouvernementalität stehen so in einem wechselseitigen Dreiecksverhältnis: >Die Anderen können indirekt gelenkt werden, die Führung wird dezentralisiert und in die Subjekte selbst verlagert. ${ }^{32}$ Das lässt sich etwa am Interesse des Staates beobachten, einen spezifischen Bevölkerungsanteil als >Muslim:innen $\mathrm{zu}$ regieren, sie als solche $\mathrm{zu}$ adressieren und $\mathrm{zu}$ erziehen, ein Interesse, das einige muslimische Akteur:innen tatsächlich aufgreifen, unter anderem auch, weil es mit ihren Interessen zusammenfällt. Amir-Moazami spricht von einem »intime[n] Verhältnis von Anerkennungsgesten und verstärkter Kontrolle der religiösen Praxis durch Sicherheitstechniken «. ${ }^{33}$ Der hegemoniale Sicherheitsdiskurs trägt dazu bei, dass Muslim:innen zu >guten (deutschen)< Staatsbürger:innen zivilisiert und in die Logik liberal-säkularer Machttechniken eingefügt werden. Demzufolge betreten, so Amir-Moazami weiter, jene Muslim:innen, die sogenannte Sicherheitspartnerschaften eingehen, die öffentliche Bühne und arbeiten der staatlichen Islamismusbekämpfung zu. Staatlich gelenkte Imamausbildungen und die Gestaltung des islamischen Religionsunterrichts begreift sie als regulative Instrumente und Kontrollmechanismen mit dem Ziel, den Islam nach christlich-säkularem Vorbild zu zähmen und europagerecht zu modernisieren. Tezcan führt das am Beispiel der Deutschen Islamkonferenz

30 Zur Rekonstruktion der Emotionen: Keskinkılıç 2021 i.E.

31 Ahmed 2018: 125.

32 Auch weitere Subjektivierungen und Positionierungen bzw. ihre Intersektionen sind wirksam, da die hegemoniale Anrede als Muslim:in nur eine Facette des Lebens eines Menschen betrifft. Im Forschungsfeld Islam/Muslim:innen in Europa liegen Arbeiten vor, die die Zusammenhänge von Macht und Subjektivierung unter verschiedenen sozialen, historischen und politischen Gesichtspunkten thematisieren u.a. als Kritik am europäischen Säkularismus (AmirMoazami 2018; Asad 2003; Mahmood 2015). 
aus, die wesentlich zur Konstruktion »eines gesellschaftsfähigen, aufgeklärten, deutschen muslimischen Subjekts « ${ }^{34}$ beigetragen hat.

Die konzeptionelle Trennung von Herrschaft und Macht, die im neuen Begriff der Gouvernementalität notwendig wird, lenkt also den Blick auf jene rassistischen Verhältnisse, die sich abseits herkömmlicher Ausschlussmechanismen ausdrücken können. Im antimuslimischen Bedrohungsszenario werden Muslim:innen unter dem Aspekt der (De-)Radikalisierung und der Prävention angesprochen, ihrer (zugeschriebenen) Zugehörigkeit nach mit Terrorismus in Verbindung gebracht und dazu angehalten, >bösen Muslim:innen $\mathrm{zu}$ entsagen. ${ }^{35}$ Einerseits greift die Disziplinarmacht durch rechtliche Normen und disziplinierende Normierungen ${ }^{36}$ weiter ein, wie zum Beispiel in Form von Anti-Terror-Gesetzen, die überwachen, bestrafen und kriminalisieren. ${ }^{37}$ Andererseits wirkt bereits das Wissen, dass die Disziplinarmacht greifen kann, disziplinierend bzw. selbstführend.

Aus dem empirischen Material der vorliegenden Studie geht hervor, dass unsere Gesprächspartner:innen ihr Verhalten nach dem Wissen, dass potentiell alle Muslim:innen betroffen sind (»wir sind auch Gefährder«), ausrichten und sich selbst kritisch befragen, ob sie als Gefahr wahrgenommen werden könnten. Sie antizipieren Rassismuserfahrungen und loten Handlungsmöglichkeiten aus, die sie nicht allzu sehr in Gefahr bringen.

Hieran setzt unser Forschungsinteresse an: Wir untersuchen die Aufforderung bzw. Anordnung, sich selbst zu führen und stellen die Frage, ob und gegebenenfalls wie das geschieht. Uns interessiert, wie es möglich ist, auf diese Aufforderung zu antworten (ohne sich gänzlich $\mathrm{zu}$ unterwerfen) und dabei eigene Interessen nicht aus dem Blick $\mathrm{zu}$ verlieren oder sogar ins Spiel $\mathrm{zu}$ bringen - also widerständig $\mathrm{zu}$ handeln. Uns interessieren in diesem Sinne, wie Gemeinden, Verbände und Vereine auf Ein- und Ausschlussmechanismen im Zusammenhang 
mit dem Sicherheitsdispositiv reagieren. Oder um es in gouvernementalitätstheoretischer Perspektive zu formulieren: Wie macht sich das muslimische Subjekt die gouvernementale Vernunft angesichts des antimuslimischen Rassismus zunutze, um Freiheiten zu erweitern und Handlungsspielräume (ob unterwerfend oder widerständig) im Spannungsverhältnis von Macht- und Selbsttechniken zu dehnen und eigene Bedürfnisse zu artikulieren? Denn wie bereits Foucault zu bedenken gibt, folgt die gouvernementale Vernunft keinesfalls starr den Staatsinteressen, sondern erwächst aus dem Zusammenspiel individueller und kollektiver Bedürfnisse und Interessen. Es gibt keine universelle Freiheit, eher »ein Verhältnis, bei dem das Maß des >zu wenig ‘ an bestehender Freiheit durch das >noch mehr $<$ an geforderter bestimmt wird «. ${ }^{38}$

\section{Subjekt, Widerstand und Handlungsmacht}

Wo Macht ausgeübt wird, gibt es auch Konflikte und Strategien, sich ihr zu widersetzen, sich aufzulehnen oder alternative Handlungsoptionen auszuloten. Um zu verstehen, wie Macht operiert, "müssen wir vielleicht die Widerstände dagegen untersuchen und die Bemühungen, diese Beziehungen aufzulösen «, schreibt Foucault. ${ }^{39}$ Mehr noch: Es gibt »keine Machtbeziehung ohne Widerstand, ohne Ausweg oder Flucht, ohne möglichen Umschwung ${ }^{40}{ }^{40}$ Das eine existiert nicht ohne das andere: Macht und Widerstand stehen »in einem Verhältnis wechselseitiger Provokation, endloser Verkettung und ständiger Verkehrung «. ${ }^{41}$

Foucault lädt dazu ein, den Gegenstand unter dem Aspekt des Widerstandes, also der Umgangsweisen und Handlungsoptionen $\mathrm{zu}$ beleuchten. Schließlich fokussiert er in seinen Studien zur Gouvernementalität keine totalitären Gesellschaften. Flügel-Martinsen vermutet,

\footnotetext{
38 Foucault 2017b: 97.

39 Foucault 2013b: 243.

40 Ebd.: 261.

41 Ebd.: 262.
} 
dass »der Prozess der politischen Subjektwerdung erst durch die interne Verschränkung von Unterwerfung und Widerstand verständlich ${ }^{42}$ wird. Beide Aspekte schließen sich weniger gegenseitig aus, als dass sie sich vielmehr wechselseitig bedingen. Der entscheidende Punkt für die Frage des Widerstands liegt darin, dass im schöpferischen Prozess der Macht Subjekte nicht einfach unterworfen, sondern hervorgebracht werden. Im Zusammenspiel von Subjektwerdung und Macht liegt ein Widerstandspotential: Die Subjekte »selbst sind es ja, die im Konflikt der Mächte mitwirken und die so, wie es erst in diachroner Perspektive sichtbar wird, die Diskurse der Macht umschreiben«. ${ }^{43}$

Den Aspekt subversiver Widerstandspraktiken im Kontext hegemonialer Anrufungen greift Butler in Haß spricht auf. Sie bekräftigt, dass Subjekte zwar diskursiv hervorgebracht werden, diese aber zeitgleich durch den Namen, mit dem sie angerufen werden, in eine Sphäre treten, in der sie den eigenen Handlungsraum entgegen des ursprünglichen Interesses der Anrede erweitern können:

»Durch den Namen, den man erhält, wird man nicht einfach nur festgelegt. Insofern dieser Name verletzend ist, wird man zugleich herabgesetzt und erniedrigt. Doch enthält der Name auch eine andere Möglichkeit, da man durch die Benennung auch eine bestimmte Möglichkeit der gesellschaftlichen Existenz erhält und erst in ein zeitliches Leben der Sprache eingeführt wird, das die ursprünglichen Absichten, die der Namensgebung zugrunde lagen, übersteigt. Während also die verletzende Anrede ihren Adressaten scheinbar nur festschreibt und lähmt, kann sie ebenso eine unerwartete, ermächtigende Antwort hervorrufen. ${ }^{44}$

42 Flügel-Martinsen 2014: 44.

43 Ebd.: 54.

44 Butler 2006: 10. 
Butler knüpft in ihren Arbeiten im Anschluss an Austin ${ }^{45}$ an Sprechakttheorien und den Performativitätsbegriff an. ${ }^{46}$ Theorien des Performativen fokussieren nicht ausschließlich das Feld der Linguistik, sondern werden »über die Sprache hinaus auf sinnenhafte Kulturphänomene aller Art übertragen «. ${ }^{47}$ Wie König bemerkt, unterscheidet sich Butlers Performativitätsbegriff grundlegend von einem theaterwissenschaftlichen Verständnis, in welchem das »)Ereignishafteく (der Aufführung) als fundamental« angesehen wird, während Butler das Prozesshafte hervorhebt. ${ }^{48}$ Butler bietet eine Perspektive an, »wie sprachliche bzw. diskursive Praxis wirklichkeitsverändernd [H.i.O.] wirken kann - nämlich

45 In How to do things with Words begreift Austin das Sprechen, etwa Beleidigungen, Befehle aber auch Ansprache und Rede, als eine Form des Handelns. Das bedeutet, die sprachliche Handlung konstituiert das, was sie äußert. Austin (1975: 109ff.) unterscheidet zwischen drei verschiedenen Sprechakten: Der lokutionäre Akt meint die Handlung, etwas zu sagen. Dazu zählen sprachliche Laute und Äußerungen nach grammatikalischen Regeln einer Sprache, die dazu dienen, Aussagen über Cegenstände und Ereignisse zu machen. Unter einem illokutionären Akt, »meaning in the traditional sense«, versteht Austin den Vollzug einer konventionellen Sprachhandlung, die sich aus einer sozialen Interaktion ergibt, also Fragen, Drohungen oder Empfehlungen. Der illokutionäre Akt vollzieht sich, indem etwas geäußert wird. Dagegen betrifft ein perlokutionärer Akt (»what we bring about or achieve by saying something«) die Folgen einer Sprachhandlung, also dadurch, dass etwas geäußert wird. Er betrifft die Wirkung, er kränkt, verunsichert oder überzeugt.

46 In Anlehnung an Austins Sprechakttheorie und unter Berücksichtigung der Foucaultschen Diskurstheorie erweitert Butler das Blickfeld um Phänomene der Identitätskonstruktionen, insbesondere im Zusammenhang mit der Performativität von Geschlecht und Sexualität: »Cender identity is performative, which means [...] that it is real only to the extent that it is performed. [...] If gender attributes, however, are not expressive but performative, then these attributes effectively constitute the identity they are said to express and reveal.« (Butler 1990: 278f.) Indem Butler auf die Rolle des Performativen in der Herausbildung von Ceschlecht verweist, weist sie Vorstellungen einer ontologischen Realität von Ceschlechtsidentität, also einer vermeintlich natürlich gegebenen, zurück.

47 Hempfer/Volbers 2011: 7.

48 König 2001: 45. 
durch die Möglichkeit der Variation oder parodistischen Subversion vorgegebener Handlung - oder Identitätsskripte «. ${ }^{49}$

Der Mechanismus lässt sich auch im antimuslimischen Rassismus beobachten. Hegemoniale Sprechhandlungen, die Muslim:innen im Akt des Bezeichnens und Besprechens als Sicherheitsbedrohung hervorbringen, können im Sinne Austins neben dem illokutionären Akt auch als perlokutionärer Akt verstanden werden. Die Diskriminierung vollzieht sich einerseits, indem antimuslimisch gesprochen wird, aber auch weil Ausschluss und Benachteiligung praktische Folgen davon sind, dass antimuslimisch gesprochen wird. Die Bezeichnung ist keine neutrale, im Gegenteil reguliert sie den Zugang zu Ressourcen, entscheidet darüber, wer (zu welchen Themen und wie) Gehör findet.

Mit dem Akt der Benennung werden Muslim:innen also dem Diskurs unterworfen und als >Muslim:innen $\prec$ hervorgebracht. Butler nennt diesen Prozess Subjektivation. Die Unterwerfung des Subjekts unter den Diskurs führt es zugleich ins Leben und ist eine Voraussetzung dafür, zu sprechen bzw. Gehör zu finden. Die Anrufung des Subjekts setzt die Bedingungen, um sich selbst im Angesicht der Macht zu konstituieren und sich Diskursregeln zu eigenen Zwecken nutzbar zu machen. Butler schreibt, »dass gerade darin, dass der herrschende, autorisierte Diskurs enteignet [H.i.O.] werden kann, eine Möglichkeit seiner subversiven Resignifikation liegt ${ }^{50}$

Das Changieren zwischen Unterwerfung und Handlungsmacht ist durchaus ambivalent zu begreifen, wie Gutiérrez Rodríguez am Beispiel (neo-)liberaler Regierungstechniken in der Ausländer-, Migrations- und Asylpolitik zeigt. Jene, die als >Migrant:innen und als >Ausländer:innen angerufen werden, werden $\mathrm{zu}$ >ethnisierten Anderen konstruiert: "Erst von dem Zeitpunkt an, an dem die Minderheiten als die rethnisch Differenten kodifiziert, kontrolliert und regiert werden, existieren sie als solche. $\aleph^{51}$ Sie werden zu defizitären Anderen konstruiert,

49 Butler 2011: 49.

50 Ebd.: 246.

51 Gutiérrez Rodríguez 2003: 171. 
in ihren Bewegungsmöglichkeiten eingeschränkt und für ihre missliche Lage selbst verantwortlich gemacht. Gleichzeitig werden sie aufgefordert, sich zu integrieren und durch Leistung und Arbeit aufzusteigen. Indem der Staat an die Individuen appelliert, entledigt er sich seiner Verantwortung. Dem Topos des homo oeconomicus unterworfen, werden Migrant:innen paradoxerweise einerseits entmächtigt und andererseits als selbstständig handelnde Subjekte adressiert. Die Anrufung als ethnisierte Andere geht in das Selbstverständnis der Individuen ein, ohne sie total zu bestimmen. Gutiérrez Rodríguez weist darauf hin, dass sich die Anrufung verkompliziert: Die Anderen gelten als Sündenbock und Problem und werden gleichzeitig »als die neuen UnternehmerInnen [H.i.O.] zelebriert ${ }{ }^{52}$ und als ökonomische Zielgruppe zur Förderung von Selbstständigkeit angesprochen. Eine direkte Analogie hierzu findet sich in der steigenden Anzahl an Förderprogrammen zur Deradikalisierung von Muslim:innen, die sich insbesondere an muslimische Selbstorganisationen richten und finanzielle Abhängigkeiten schaffen. ${ }^{53}$ Die Biopolitik bindet >die Anderen in die neoliberale Logik ein, vermag sie aber nicht vollständig zu kontrollieren:

»Denn der Versuch der Domestizierung der Subjekte scheitert an der Hartnäckigkeit der unbestimmbaren und ungezügelten kollektiven Praxis. Die staatlichen Führungsprogrammatiken im Sinne der Couvernementalität mögen daher aus den Praktiken und Rationalitäten der Subjekte schöpfen, können diese aber nicht vollständig abbilden und kontrollieren. Die Praxis der Subjekte ist nicht zu fixieren, sie durchdringt die Risse der Strukturen, in denen sie zwar verfangen, aber auch zugleich beweglich ist. Das Konzept der Gouvernementalität wirft daher spannende Fragen bezüglich der Vereinnahmung subjektiver Praktiken und Denkformen durch staatliche Institutionen auf. Zugleich verweist es auf die Grenzen dieser Führungslogik, die an

\footnotetext{
52 Ebd.: 176.

53 Attia 2019b; Qasem 2019.
} 
der Praxis und dem widerspenstigen Begehren der Subjekte, die sie zu modellieren sucht, scheitert. ${ }^{54}$

Ähnlich argumentiert auch Rose. Mit Foucault und Butler definiert sie widerständiges Handeln als »eine Praxis der Grenzbearbeitung, eine Praxis des kritischen Fragens und einer kritischen Haltung, die genau jene bislang gültigen Grenzziehungen zwischen dem >Normalen dem >Verwerflichen zum Gegenstand der Auseinandersetzung macht, indem sie ihnen nur begrenzt entspricht ". ${ }^{55}$ Auch Wagenknecht befragt die widersprüchlichen und ambivalenten Verhaltensweisen im Umgang mit hegemonialer Anrede und lenkt dazu den Blick auf die in der Lebenspraxis von Subjekten gesammelten Erfahrungen und Bedürfnisse, »die Ausgangspunkt für die Überwindung herrschaftlicher Zurichtung sein können. $\ll^{56}$ Die Möglichkeit, den Namen zurückzuweisen und das Selbst abseits der diskursiven Anrufung zu gestalten, gelingt, indem Ambivalenz produziert wird. Die Anrufung eines Subjekts kann auf eine Weise beantwortet werden, die die Ansprache irritiert. Sie vermag es, die hervorgebrachte Repräsentation, unter der das Subjekt benannt wird, zu stören und zu brechen.

»Die verschiedenen ökonomischen, politischen, ideologischen, kulturellen usw. Prozesse der Ambivalenzproduktionen gehören zu einer wesentlichen Veränderung des gesellschaftlichen Ganzen, zur Formierung einer neuen Produktionsweise. [...] Die Ambivalenz entsteht als Veränderung in der Artikulation gesellschaftlicher Widersprüche, in der Art und Weise, wie sie verbunden sind und sich gegenseitig formen. Vormals klare Differenzlinien werden verwischt, werden nicht nur durch ideologische Rede vernebelt, sondern tatsächlich uneindeutig. ${ }^{57}$

\footnotetext{
54 Gutiérrez Rodríguez 2003: $176 f$.

55 Rose 2015: 332.

56 Wagenknecht 2003: 200.

57 Ebd.: 213.
} 
Flügel-Martinsen bringt es folgendermaßen auf den Punkt: »Widerstand bedeutet so gesehen Bedeutungsverschiebung «. ${ }^{58}$

Aus der Skepsis gegenüber metaphysisch überhöhten Begrifflichkeiten und absoluten Wahrheiten, die Foucaults Arbeiten durchziehen, warnt Flügel-Martinsen davor, eine systematische Theorie des Widerstandes bei Foucault zu suchen, und plädiert stattdessen dafür, die Frage nach widerständigem Handeln umzudeuten:

» $Z u$ fragen ist also nicht, wie Subjekte generell Widerstand gegen Machtprozesse, die sie konstituieren, leisten können, sondern zu untersuchen sind die je spezifischen Modalitäten der Subjektkonstitution und des Widerstands in konkreten historischen Konstellationen ${ }^{59}$

Jain geht davon aus, dass die Ambivalenz und Widersprüchlichkeit von Wünschen und Begierden eine Reflexion ermöglichen, die wiederum Widerspruch und infolge dessen auch »die Spannung zwischen Subjekt und Politik, die die eigentliche Grundlage des politischen Verhältnisses ist, wieder beleben $«{ }^{60}$ Er sieht in der Entwicklung eines solchen Subjektbewusstseins die notwendige Voraussetzung für kollektive politische Handlungsfähigkeit. Das Subjekt muss

»[...] sich selbst als solches erkennen und begreifen und sich zugleich (kritisch und widerständig) auf seine Umwelt beziehen. Es muss ein sauthentisches< Bewusstsein seiner Selbst entwickeln, und dies setzt eine (Selbst-) Reflexion voraus, die gerade für die Widersprüche und die Ambivalenz des Seins und des Selbst offen ist. Ein solcher reflexiver Authentizitätsbegriff bedeutet, dass das Selbst seine immanente, nicht transzendierbare innere Widersprüchlichkeit und Ambivalenz entdeckt, um seinen Widerspruch für die Möglichkeit des Widersprüchlichen und des Differenten zu entfachen. Die reflexive Authentizität des Selbst bestünde also gerade in der Spiegelung und im Zu-

\footnotetext{
58 Flügel-Martinsen 2014: 54.

59 Ebd.: 56.

60 Jain 2003: 255.
} 
lassen von innerer wie äußerer Ambivalenz - nicht im Beharren auf Eindeutigkeit und Identität. « ${ }^{61}$

Das Ambivalenz-Dispositiv, das in diesen Überlegungen als widerständige Reaktion auf eine unterwerfende Anrede bemüht wird, lenkt den Blick auf vielfältige Strategien, mit denen auch Muslim:innen auf den antimuslimischen Sicherheitsdiskurs antworten. Butlers Subjektivationstheorie gemäß werden sie als (Gefahren-)Subjekt angerufen und hervorgebracht, ihnen wird ein Name und damit einhergehend eine gesellschaftliche Rolle zugewiesen, die sie ins Leben führt und zugleich die Bedingungen schafft, um handlungsfähig und gehört zu werden. Themen, die an sie herangetragen werden - Salafismus, Terrorismus, (De-)Radikalisierung und Prävention - können als Muslim:innen Angerufene aufgreifen, sich $\mathrm{zu}$ ihnen positionieren und sich mit ihnen auseinandersetzen und zugleich eigene Erfahrungen, Interessen und Bedürfnisse artikulieren.

Das erlaubt, islambezogene Kontrastfolien $\mathrm{zu}$ irritieren und um andere Identifikationspunkte $\mathrm{zu}$ erweitern, unter denen sich die jeweiligen Lebensentwürfe und -realitäten gestalten, Erfahrungen mit dem antimuslimischen Rassismus zu artikulieren und das muslimische Selbst $\mathrm{zu}$ verkomplizieren, statt Identität $\mathrm{zu}$ vereinheitlichen. Essentialistische Anrufungen als >Muslim:in können zurückgewiesen (und damit verweigert) werden, sie können unter Berücksichtigung verschiedener religiöser, säkularer, politischer, sozialer und vergeschlechtlichter Deutungsmuster differenziert und multipliziert werden, sodass (nicht nur individuell) Ambivalenz produziert wird. Die »Praxen dieser Überwindung [sind] notwendig kollektive Praxen ${ }^{62}$, gerade weil Erfahrungen des antimuslimischen Rassismus strukturell eingeschrieben sind und im größeren sozialen Zusammenhang existieren. (Als) Muslim:innen (Markierte) sind sich der kollektiven Erfahrungsdimension bewusst. Sie verschaffen sich gegenseitig Gehör,

61 Ebd.: $242 f$.

62 Wagenknecht 2003: 200. 
beziehen sich aufeinander und denken gemeinsam konkrete Utopien. Die Erfahrung von `Gleichheit kann mit einer »Solidarität der Verschiedenheiten ${ }^{63}$ verknüpft werden.

\section{Schlussfolgerung}

In der Auseinandersetzung mit dem antimuslimischen Rassismus kommt man nicht umhin, dem zentralen Stellenwert des hegemonialen Sicherheitsdiskurses Rechnung zu tragen. Wie aus dem empirischen Material der vorliegenden Studie hervorgeht, sind unsere muslimischen Gesprächspartner:innen in ihrem Alltag und in ihrem Beruf, in öffentlichen Institutionen und auf offener Straße, durch mediale und politische Ansprache mit Misstrauen und Verdacht konfrontiert. Sie müssen sich verantworten und Stellung beziehen, sich zu >islami(sti)scher Gewalt positionieren, sich distanzieren und als 'guter Ausnahmen in Szene setzen. Die Erfahrung, ihrer (vermeintlichen) Religion, Kultur und Herkunft nach kollektiv als Gefahr wahrgenommen zu werden, hat Einfluss auf ihre Gefühle und ihr Selbstbild, darauf, an welchen Orten sie Präsenz zeigen und welche sie wiederum meiden, ob und wie sie Religiosität praktizieren, wie sie sich kleiden, was und wie sie (be-)sprechen oder (ver-)schweigen. Doch Muslim:innen sind wie andere auch - keine passiven Objekte einer allgegenwärtigen und omnipotenten Macht.

Hieran knüpft unsere Studie an: Wir fragen danach, wie das muslimische Subjekt Deutungs- und Handlungsmacht (zurück-)erlangt. Vor dem Hintergrund von Foucaults Ausführungen zum Verhältnis von Sicherheit, Bevölkerung und Gouvernementalität sowie seinem Diktum, dass es dort, wo es Macht gibt, auch Widerstand gibt, fragen wir danach, wie die als Muslim:innen Angerufenen das Verhältnis von Unterwerfung und Widerstand angesichts des Sicherheitsdiskurses austarieren. Uns stellt sich also die Frage nach den Spielräumen: Wie navigieren Muslim:innen durch Strukturen der Verbesonderung 
und Diskriminierung, durch Ein- und Ausschlüsse, durch Stigmatisierung und Generalverdacht? Wie knüpfen sie an gesellschaftliche Debatten um Sicherheit an, wie beteiligen sie sich an Aushandlungsprozessen und wie positionieren sie sich, um sich Gehör für ihre Erfahrungen, Anliegen und Bedürfnisse zu verschaffen? Wie gelingt es >Muslim:innen am Diskurs teilzuhaben und dabei Definitions- und Handlungsmacht (wieder) zu gewinnen? Dabei interessieren uns auch widersprüchliche und uneindeutige Gleichzeitigkeiten von Unterwerfung und Widerstand, etwa wie in der affirmativen Selbstführung auch seigene ‘ Interessen artikuliert und verfolgt werden können. Ausgehend davon, dass im Machtverhältnis Aushandlungspotential liegt und in der Selbstführung Interpretations- und Handlungsspielräume genutzt werden können, ist es also möglich, dass sich Muslim:innen im Prozess und in der Aneignung der sicherheitspolitischen Selbstführung nicht nur anpassend unterwerfen, sondern im Zuge ihrer Antwort den Sicherheitsdiskurs verschieben und ihn nutzen, um anderen Interessen nachzugehen als jenen, die von ihnen erwartet werden.

Schließlich lässt sich im empirischen Material ein weites Archiv an widerständigen Selbstbildern, Verhaltensweisen und alternativen Handlungsoptionen nachvollziehen, die das Gegenstück zur antimuslimischen Regierungskunst der Gouvernementalität bilden. Um es in Foucaults Worten $\mathrm{zu}$ sagen, geht es um »die Kunst nicht regiert zu werden bzw. die Kunst nicht auf diese Weise und um diesen Preis regiert zu werden «. ${ }^{64}$ Unsere Gesprächspartner:innen sind darum bemüht, zwischen verschiedenen gesellschaftlichen Rollen $\mathrm{zu}$ changieren und sie miteinander in Dialog zu bringen. Sie berichten von Rassismuserfahrungen, setzen sie in einen politischen, historischen Zusammenhang und untermauern zugleich professionelle, wissenschaftliche und aktivistische Sprechpositionen. Sie ordnen und verkomplizieren gesellschaftliche Themen, die an sie herangetragen werden, sie weiten Fragestellungen aus, analysieren und kontextualisieren eigene Positionierungen, differenzieren Diskussionsräume aus und untergraben hegemoniale Sprechakte.

64 Foucault 1992: 12. 
Insgesamt fordern sie defizitäre Islamdebatten heraus, irritieren homogene Kategorien und rücken komplexe Lebensrealitäten ins Feld, indem sie auf globalhistorische, queer-feministische, unterschiedlich religiöse, säkulare und weitere Erfahrungen und Erinnerungen zurückgreifen. Sie sind sich der Ausschlussmechanismen des Sicherheitsdiskurses bewusst, reflektieren Anforderungen nach Prävention und Deradikalisierung unter dem Aspekt des antimuslimischen Rassismus und rücken eigene Sicherheitsbedürfnisse und staatsbürgerliche Interessen ins Zentrum, die sie den Medien, der Politik und der Gesamtgesellschaft gegenüber geltend machen. Sie kritisieren ein Klima des Misstrauens, der Verdächtigungen und Konkurrenzen, das Einfluss auf die Community und die Zusammenarbeit untereinander nimmt, und reflektieren angesichts dessen Möglichkeiten der Solidarisierung. Und sie tauschen Visionen für eine bessere, community-nahe Zusammenarbeit aus, in der sie nicht gegeneinander ausgespielt werden, sondern stattdessen Räume zur Selbstermächtigung gestalten. 